\title{
SCIENTIFIC REPORTS

\section{OPEN Corticosteroid eye drop instillation aggravates the development of Acanthamoeba keratitis in \\ rabbit corneas inoculated with Acanthamoeba and bacteria}

Received: 28 March 2019

Accepted: 16 August 2019

Published online: 06 September 2019

\author{
Hayate Nakagawa ${ }^{1}$, Naohito Koike ${ }^{2}$, Tomoko Ehara ${ }^{2}$, Takaaki Hattori ${ }^{1}$, Akitomo Narimatsu ${ }^{1}$, \\ Shigeto Kumakura ${ }^{1} \&$ Hiroshi Goto $^{1}$
}

The role of topical corticosteroids in management of Acanthamoeba keratitis (AK) remains controversial. Using a rabbit AK model, we investigated whether corticosteroid use is a risk factor of AK. Acanthamoeba $\left(1 \times 10^{5} / \mathrm{ml}\right)$ was incubated with two densities of $P$. aeruginosa (PA; high-PA: $1 \times 10^{8} /$ $\mathrm{ml}$, low-PA: $3 \times 10^{5} / \mathrm{ml}$ ) before corneal inoculation. Rabbit corneas were inoculated with Acanthamoeba alone or Acanthamoeba plus PA and administered levofloxacin and betamethasone sodium phosphate (BSP) eye drops for 5 or 7 days. Infected rabbit eyes were evaluated for clinical score and Acanthamoeba by histological examination. Acanthamoeba alone and BSP treatment did not produce keratitis. Corneas inoculated with Acanthamoeba plus low-PA treated immediately with levofloxacin and BSP remained clear with few infiltrates. Corneas inoculated with Acanthamoeba plus low-PA treated with levofloxacin immediately and BSP $12 \mathrm{~h}$ later developed severe keratitis. Corneas inoculated with Acanthamoeba plus high-PA treated immediately with levofloxacin and BSP also developed severe keratitis. Acanthamoebae were detected by PAS staining in corneas inoculated with Acanthamoeba plus high-PA treated with levofloxacin and BSP. Topical corticosteroids have the potential to aggravate AK when cornea is infected by Acanthamoeba with a critical number of bacteria or when corticosteroids are given after infection has established by Acanthamoeba with small number of bacteria.

Acanthamoeba keratitis (AK) is a rare but severe corneal infection. This painful, sight-threatening, and difficult-to-treat corneal infection is caused by an opportunistic protist belonging to the genus Acanthamoeba $a^{1-3}$. The treatment of $\mathrm{AK}$ has not been satisfactory and requires improvement ${ }^{4}$. Topical corticosteroids are frequently needed to control pain and inflammation in $\mathrm{AK}^{5}$. However, the role of topical corticosteroids in the management of AK remains controversial ${ }^{6-9}$. Robaei et al. ${ }^{8}$ reported that corticosteroid use before diagnosis of AK is highly predictive of poorer visual outcome. McClellan et al. ${ }^{9}$ also found that exposure of Acanthamoeba trophozoites and cysts to dexamethasone increases the pathogenicity of the organisms. From these reports, corticosteroid use may be a risk factor for the development or progression of $\mathrm{AK}$. We therefore investigated whether corticosteroid use is a risk factor of AK in vivo; and if it is a risk factor, how corticosteroids affect the pathology of AK.

Recently, much interest has been focused on the bacterial symbionts extracted from Acanthamoeba strains isolated from patients with $\mathrm{AK}^{10-13}$. We have investigated the relationship between bacteria and Acanthamoeba in the development of keratitis using a rabbit $\mathrm{AK}$ model to reveal the pathogenic mechanisms of $\mathrm{AK}^{14,15}$. Our previous investigations showed that the presence of bacteria may be indispensable and a critical number of bacteria with adequate time for Acanthamoeba-bacteria interaction may be required for the development of AK. Our previous experimental AK animal model using a clinical isolate of Acanthamoeba strain and E. coli showed the development of severe keratitis ${ }^{14}$. Moreover, the Acanthamoeba strain ATCC 50492 and P. aeruginosa strain PAO-1 caused keratitis that peaked at days 1-2 and thereafter attenuated gradually ${ }^{15}$. It remains unclear why the

${ }^{1}$ Department of Ophthalmology, Tokyo Medical University, Shinjuku City, Japan. ${ }^{2}$ Department of Microbiology, Tokyo Medical University, Shinjuku City, Japan. Correspondence and requests for materials should be addressed to H.N. (email: rocklife1031@yahoo.co.jp) 
A

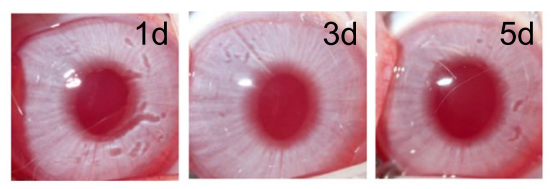

$\mathrm{B}$

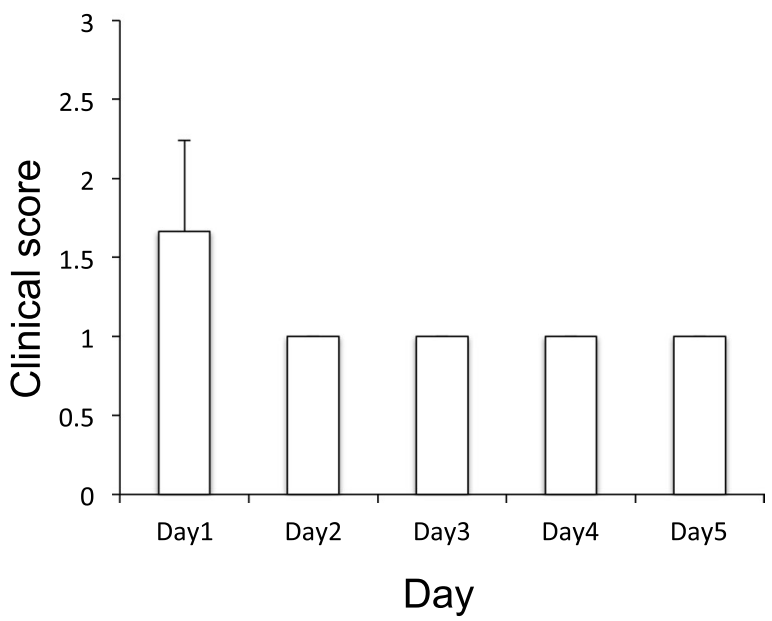

Figure 1. Clinical results of rabbit corneas inoculated with Acanthamoeba alone in which BSP eye drops three times daily was started immediately after inoculation. (A) Representative rabbit corneal photographs on days 1-5 after inoculation with Acanthamoeba alone and administration of BSP eye drops 3 times a day. The corneas remain clear. (B) Rabbit corneas inoculated with Acanthamoeba alone and treated with BSP eye drops shows few infiltrates and mild inflammation in the cornea.

development of AK weakened. We speculated that P. aeruginosa is not capable of accelerating the development of $\mathrm{AK}$, and that the bacterial species and the interaction with Acanthamoeba may be important for the development of AK.

In the present study, we investigated the effect of betamethasone sodium phosphate (BSP) eye drop in a rabbit AK model produced by inoculation of Acanthamoeba castellanii ATCC 50492 and P. aeruginosa strain PAO-1. We also investigated the interaction between the two organisms in the presence of corticosteroids.

\section{Results}

Inoculation of acanthamoeba alone and treatment with BSP eye drops did not induce keratitis. We examined whether inoculation of the Acanthamoeba strain (ATCC 50492) alone followed by treatment with BSP eye drops induces keratitis. After rabbit corneas were inoculated with Acanthamoeba, BSP eye drops 3 times a day were started immediately. The corneas were examined microscopically after inoculation on day 0 until day 5. Rabbit corneas inoculated with Acanthamoeba and treated with BSP eye drops showed few infiltrates on day 1 post-infection (Fig. 1A,B). The corneas remained clear during the experimental period. Based on our definition of clinical end point, the rabbits inoculated with Acanthamoeba were euthanized on day 5.

Antibiotic and corticosteroid treatment of corneas inoculated with acanthamoeba plus low- density P. aeruginosa. In our previous study, corneas inoculated with a mixture of Acanthamoeba plus low-density $P$. aeruginosa and treated immediately with LVFX eye drops showed few infiltrates on day 1 post-infection and remained clear during the experiment. To investigate the effect of corticosteroids, we used a similar experimental infection model and compared the clinical scores of keratitis produced by Acanthamoeba plus $P$. aeruginosa treated with LVFX with or without corticosteroid eye drop. We treated rabbit corneas with $1.5 \%$ LVFX eye drops or 1.5\% LVFX eye drops and BSP eye drops immediately after inoculation of Acanthamoeba plus low-density $P$. aeruginosa. The corneas in both groups showed few infiltrates on day 1 post-infection and remained clear during the experiment (Fig. 2A,B). There were no differences in clinical score between the two groups during the observation period (Fig. 2D).

We also investigated how corticosteroid affects the cornea in which Acanthamoeba infection has already established. Corneas inoculated with Acanthamoeba plus low-density P. aeruginosa were treated with $1.5 \%$ LVFX eye drops immediately followed by BSP eye drops $12 \mathrm{~h}$ after inoculation. These corneas showed few infiltrates on day 1 post-infection, but developed mild infiltrates at the center of the cornea with diffuse corneal edematous on day 3 post-infection, and progressed to severe keratitis on day 5 post-infection (Fig. 2C).

Keratitis was significantly more severe in the group treated with BSP eye drops after a lapse of $12 \mathrm{~h}$ following inoculation compared with the other two groups from day 3 to day 5 (Fig. 2D). 


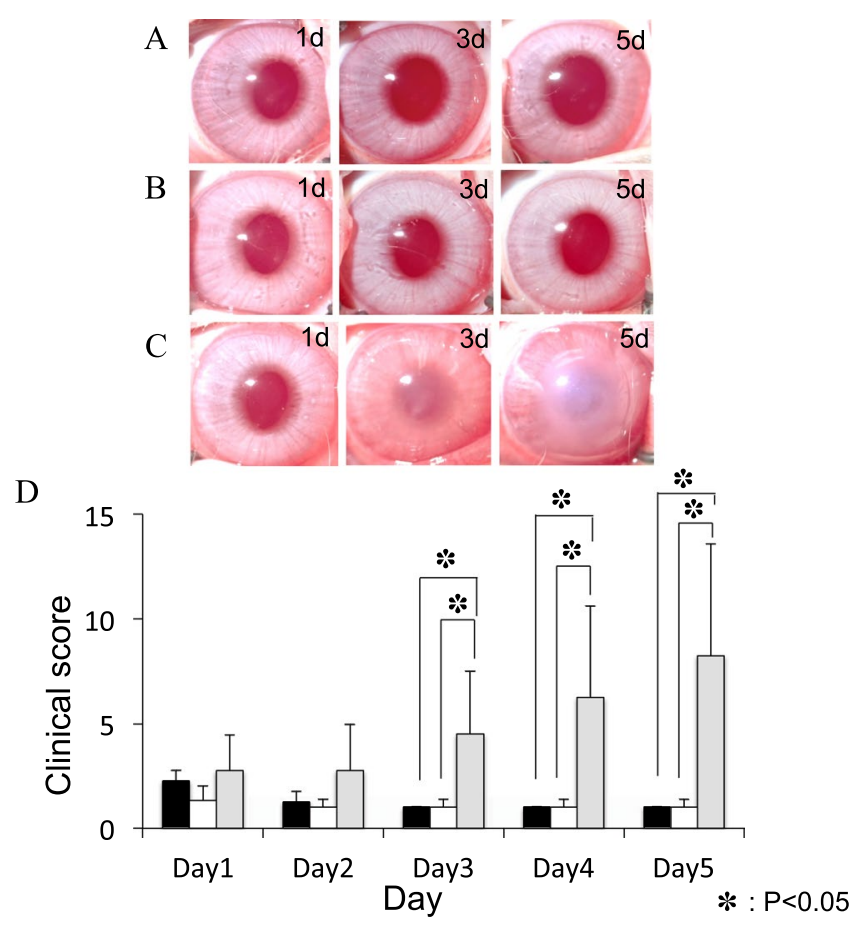

Figure 2. Clinical results of treatment with LVFX eye drop alone or with LVFX and BSP eye drops for keratitis produced by inoculating Acanthamoeba with a low density of $P$. aeruginosa. (A-C) Representative photographs (days $0-5$ ) of keratitis in rabbit eyes inoculated with Acanthamoeba $\left(1 \times 10^{5} / \mathrm{ml}\right)$ plus low-density P. aeruginosa $\left(3 \times 10^{5} / \mathrm{ml}\right)$ followed immediately by treatment with $1.5 \%$ LVFX eye drops 3 times a day (A), or with $1.5 \%$ LVFX eye drops together with BSP eye drops 3 times a day (B), or immediately with $1.5 \%$ LVFX eye drops and $12 \mathrm{~h}$ later with BSP eye drops, both 3 times a day (C). (D) Clinical scores (days 1-5 post-inoculation) of keratitis in the above three groups. Closed bar denotes treatment immediately after inoculation with $1.5 \%$ LVFX eye drops; open bar denotes treatment immediately after inoculation with 1.5\% LVFX + BSP eye drops; and gray bar denotes treatment immediately after inoculation with $1.5 \%$ LVFX followed by BSP eye drops $12 \mathrm{~h}$ later. Keratitis is significantly more severe in the group treated with BSP eye drops after a delay following inoculation compared to the other two groups. ${ }^{*} \mathrm{P}<0.05, \mathrm{n}=4$ in each group.

Antibiotic or antibiotic and corticosteroid treatment of corneas inoculated with acanthamoeba plus high-density P. aeruginosa. In our previous study ${ }^{15}$, corneas inoculated with Acanthamoeba pre-incubated with a high density of P. aeruginosa and treated with LVFX developed severe keratitis that peaked at days 1-2 and thereafter attenuated gradually. We investigated the effect of corticosteroid using a similar keratitis model. Rabbit corneas inoculated with Acanthamoeba plus high-density P. aeruginosa treated immediately after inoculation with LVFX eye drops showed infiltration in the cornea and anterior chamber inflammation on days 1-2 post-infection. On day 3, infiltration in the central cornea was attenuated slightly and corneal edema was reduced. On day 5, corneal infiltration and corneal opacity were further reduced. (Fig. 3A). Consistent with previous report, the keratitis peaked at days 2 and thereafter attenuated gradually. On the other hand, rabbit corneas inoculated with Acanthamoeba plus high-density P. aeruginosa treated with LVFX eye drops and BSP eye drops developed severe keratitis (Fig. 3B). These eyes showed diffuse infiltration and edema in the cornea together with anterior chamber inflammation on days 1-3 post-infection. On day 5-7, diffuse infiltration in the cornea and corneal edema aggravated. On day 7 , corneal infiltration further increased and corneal melting was observed. Significant differences in clinical score were observed between LVFX eye drops alone and LVFX with BSP eye drops from day 3 to day 7 (Fig. 3C).

Pathological evidence of the presence of acanthamoeba in cornea tissue. We performed histopathological study to examine the existence of Acanthamoeba in rabbit corneas inoculated with Acanthamoeba plus high-density P. aeruginosa treated with LVFX and BSP eye drops. Initially, we used Fungiflora Y staining to detect Acanthamoeba cyst, but no Acanthamoeba cysts were detected in corneal tissue using this method, consistent with our previous finding ${ }^{14}$. Next, we performed PAS staining. Structures similar to Acanthamoeba cysts were detected in central corneal stroma (Fig. 4A). These structures were round, similar in shape to Acanthamoeba cysts, but without the specific double-wall structure of the Acanthamoeba cysts. The acanthamoebae were surrounded by phagocytes of the rabbit (Fig. 4B) and aggregated in central corneal stroma (Fig. 4C). We also investigated the histopathological findings of the corneas inoculated with Acanthamoeba plus low-density P. aeruginosa treated with levofloxacin immediately and with BSP 12 hours later. By PAS staining, we found no aggravation of acanthamoebae in corneal stroma, in contrast to the findings in corneas inoculated with Acanthamoeba plus high-density $P$. aeruginosa treated with BSP immediately. In histopathological examination, we found only mild corneal infiltration (Supplemental Fig. 1). 
A
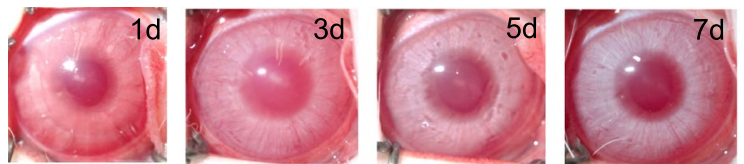

$\mathrm{B}$
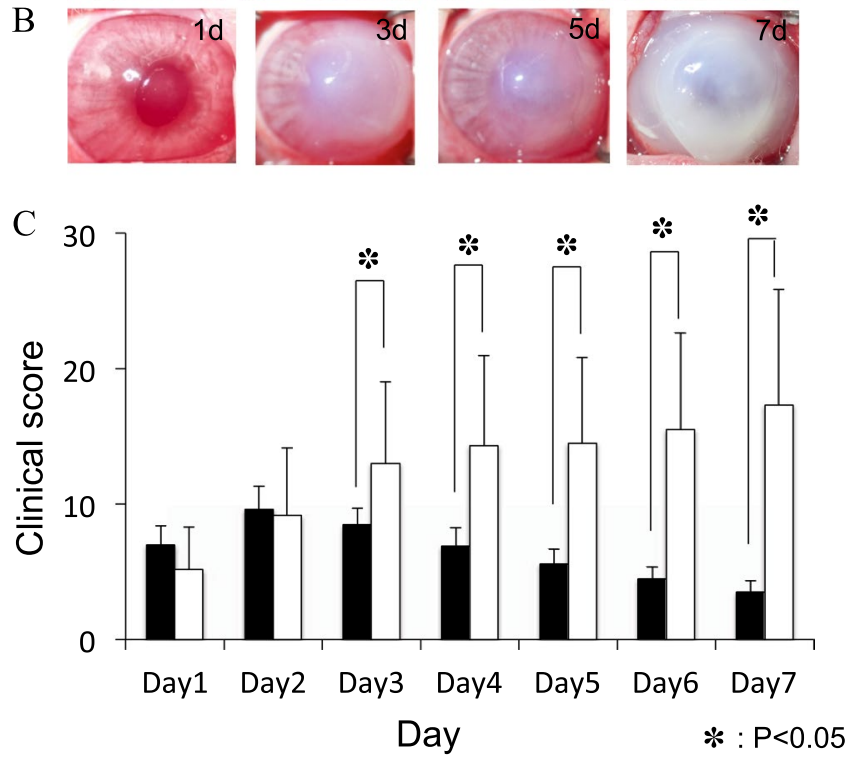

Figure 3. Clinical results of treatment with LVFX eye drop alone or with LVFX and BSP eye drops for keratitis produced by inoculating Acanthamoeba with a high density of $P$. aeruginosa. (A,B) Representative photographs of keratitis in rabbit eyes inoculated with Acanthamoeba $\left(1 \times 10^{5} / \mathrm{ml}\right)$ plus high-density P. aeruginosa $\left(1 \times 10^{8} /\right.$ $\mathrm{ml}$ ) and treated immediately with 1.5\% LVFX eye drops 3 times a day (A), or with 1.5\% LVFX eye drops and BSP eye drops 3 times a day (B). (C) Clinical scores (days 1-5 post-inoculation) of keratitis in the above two groups. Closed bar denotes treatment immediately after inoculation with $1.5 \%$ LVFX eye drops; and open bar denotes treatment immediately after inoculation with 1.5\% LVFX + BSP eye drops. Clinical scores are significantly higher in inoculated corneas treated with LVFX and BSP compared with LVFX alone. ${ }^{*} \mathrm{P}<0.05$, $\mathrm{n}=4$ in each group.

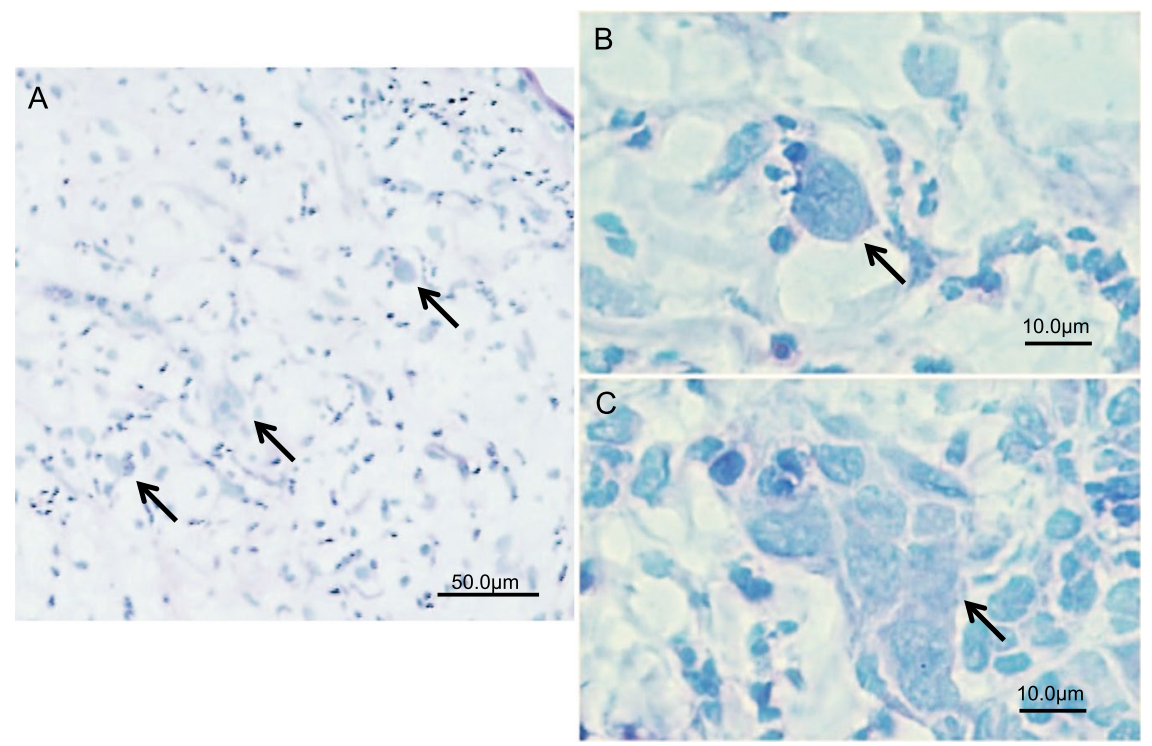

Figure 4. Histopathological evaluation by PAS staining of frozen sections of cornea inoculated with Acanthamoeba and a high density of $P$. aeruginosa followed by treatment with $1.5 \%$ LVFX and BSP eye drops. (A) Acanthamoebae (arrows) are observed in corneal stromal tissue under low magnification. (B,C) Under high magnification, structures resembling Acanthamoeba cysts are observed. In (B), Acanthamoeba cyst (arrow) is surrounded by phagocytes. In (C), aggregation of acanthamoebae (arrow) is observed. 


\section{Discussion}

Our results showed that corneal inoculation of the Acanthamoeba strain (ATCC 50492) alone followed by treatment with BSP eye drops did not cause keratitis. Our previous report showed that a clinical isolate of Acanthamoeba strain alone without bacteria or a standard Acanthamoeba strain (ATCC 50492) alone did not induce keratitis in animal models ${ }^{14,15}$. Taken these results together, Acanthamoeba in the absence of symbionts may not be capable of producing keratitis regardless of the absence or presence of corticosteroids.

We evaluated the effects of topical corticosteroids on the development of AK in corneas inoculated with Acanthamoeba pre-incubated $(2 \mathrm{~h})$ with two densities of $P$. aeruginosa. Keratitis did not develop when corneas inoculated with Acanthamoeba plus low-density P. aeruginosa were treated immediately with $1.5 \%$ LVFX eye drops and BSP eye drops 3 times a day. The corneas remained almost clear during the experiment. On the other hand, severe keratitis developed when corneas inoculated with Acanthamoeba plus low-density P. aeruginosa were treated with $1.5 \%$ LVFX eye drops immediately after inoculation and additionally with BSP eye drops after a delay of $12 \mathrm{~h}$. This result suggests that the pathological potential of Acanthamoeba is enhanced leading to the development of severe keratitis when topical corticosteroids are administered after Acanthamoeba infection has already established in the cornea. McClellan et al. ${ }^{9}$ established an AK animal model using Chinese hamsters and compared the severity of keratitis with and without systemic dexamethasone administration. Keratitis was more severe in animals treated with dexamethasone compared with untreated controls. The investigators administered dexamethasone to Chinese hamsters $24 \mathrm{~h}$ after corneal infection by Acanthamoeba. Despite the difference in experimental model, their study and ours both demonstrate that delayed treatment with corticosteroids increases the severity of keratitis in AK. We speculate that the cornea inoculated with Acanthamoeba plus low-density $P$. aeruginosa and treated immediately with LVFX develops mild keratitis, which may then regress because the pathological potential of Acanthamoeba is not adequate to accelerate corneal inflammation. On the other hand, topical corticosteroids administered $12 \mathrm{~h}$ after inoculation when Acanthamoeba infection has already established in the cornea may enhance the pathogenicity of Acanthamoeba to accelerate corneal inflammation.

Rabbit corneas inoculated with Acanthamoeba plus high-density P. aeruginosa and treated immediately with LVFX eye drops and BSP eye drops also developed severe keratitis resulting in corneal melting. In our previous study, the same animal model treated with LVFX but no BSP eye drops developed keratitis that peaked at day 2 and thereafter attenuated gradually ${ }^{15}$. The findings of our two studies suggest that Acanthamoeba pre-incubated with $P$. aeruginosa exceeding a critical number effectively establishes infection in the cornea promptly after corneal inoculation, and under such condition, exposure of Acanthamoeba to corticosteroids increases the pathogenicity of the organism accelerating the progression of corneal inflammation to a fulminant state.

In our previous study, we investigated the interaction of Acanthamoeba and bacteria in the development of $\mathrm{AK}$, and showed the importance of the number of bacteria co-inoculated with Acanthamoeba in the development of $\mathrm{AK}^{15}$. In the present study, we found that the number of bacteria co-inoculated with Acanthamoeba also affected the effect of corticosteroids on the development of AK. When corneas are infected with Acanthamoeba and a low density of bacteria, the timing of administering topical corticosteroids may be important for the development of keratitis. We therefore recommend to administer antibiotic eye drops to reduce the number of bacteria as a measure to prevent severe $\mathrm{AK}$ in patients who are currently treated with topical corticosteroids.

In a study by McClellan et al. ${ }^{9}$, dexamethasone-treated trophozoites or cysts induced a significant cytopathic effect in corneal epithelial cells compared with untreated organisms. Their study indicates that corticosteroids may change the pathological potential of Acanthamoeba, which was observed in our in vivo study. Some studies suggest that corticosteroids inhibit encystment of Acanthamoeba trophozoites ${ }^{7}$, and that trophozoite proliferation is accelerated when trophozoites are treated with corticosteroids ${ }^{9}$. These studies imply that administration of corticosteroids may increase the ratio of trophozoites in AK. Our histopathological examination also showed that Fungiflora-Y staining detected no Acanthamoeba cysts, whereas PAS staining detected structures similar to Acanthamoeba cysts but lacked the specific double-wall structure of Acanthamoeba cysts. Our histopathological study thus suggest that corticosteroids may inhibit encystment of Acanthamoeba trophozoites. In a study by Garate et al. ${ }^{16}$, the pathogenic potential of Acanthamoeba correlates directly with the expression level of the parasite mannose-binding protein (MBP). Acanthamoeba cysts express markedly reduced levels of MBP compared with Acanthamoeba trophozoites. Extrapolating this finding to the results of our pathological examinations, we speculate that corticosteroids may induce transformation of the pathogenicity of Acanthamoeba in vivo, inhibiting encystment of Acanthamoeba trophozoites and upregulating the expression level of the MBP.

The present study has a limitation. The rabbit AK model and human AK differ in The mechanism of Acanthamoeba infection. In our model, Acanthamoeba is inoculated directly into corneal stromal tissues to produce severe keratitis. The merit of this animal model is the high efficiency of establishing keratitis and simple evaluation of inflammation. However, specific findings of human AK cannot be observed.

In conclusion, our study demonstrated that topical corticosteroids aggravated AK in rabbit corneas inoculated with Acanthamoeba pre-incubated with P. aeruginosa. Use of topical corticosteroids potentially leads to development of severe AK when administered immediately after the cornea is infected with Acanthamoeba containing a critical number of bacteria or when administered after a delay when Acanthamoeba containing a small number of bacteria has already established infection in the cornea. If topical corticosteroids have to be used in eyes affected by $\mathrm{AK}$, administration of antibiotic eye drops to reduce the number of bacteria may be useful to prevent severe AK.

\section{Materials and Methods}

Cell cultures. All studies were performed using a human ocular isolate of Acanthamoeba castellanii obtained from the American Type Culture Collection (ATCC 50492). The Acanthamoeba strain was maintained in PYG medium. A basal medium ( $20 \mathrm{~g}$ of proteose peptone and $1.0 \mathrm{~g}$ of yeast extract in $900 \mathrm{ml}$ of distilled water) and a $2 \mathrm{M}$ glucose stock solution ( $18 \mathrm{~g}$ of glucose and $1.0 \mathrm{~g}$ of sodium citrate $2 \mathrm{H}_{2} \mathrm{O}$ in $50 \mathrm{ml}$ of distilled water) were first 
prepared. Then, PYG medium was prepared by mixing $900 \mathrm{ml}$ of basal medium, $10 \mathrm{ml}$ of $0.4 \mathrm{M} \mathrm{MgSO}_{4}, 8 \mathrm{ml}$ of $0.05 \mathrm{M} \mathrm{CaCl}_{2}, 10 \mathrm{ml}$ of $0.005 \mathrm{M} \mathrm{Fe}\left(\mathrm{NH}_{4}\right)_{2}\left(\mathrm{SO}_{4}\right)_{2} \bullet 6 \mathrm{H}_{2} \mathrm{O}, 10 \mathrm{ml}$ of $0.25 \mathrm{M} \mathrm{KH}_{2} \mathrm{PO}_{4}$ and $50 \mathrm{ml}$ of $2 \mathrm{M}$ glucose stock solution. The Acanthamoeba cells were resuspended in fresh PYG medium prior to experiments. Pseudomonas aeruginosa (PAO-1) was cultured on heart infusion agar for $24 \mathrm{~h}$ at $35^{\circ} \mathrm{C}$, and suspended in PYG medium for use. Based on minimum inhibitory concentration (MIC) test performed by the broth microdilution method as described by the CLSI guideline ${ }^{17}$, Pseudomonas aeruginosa PAO- 1 was susceptible to $1 \mu \mathrm{g} / \mathrm{ml}$ of levofloxacin (LVFX; Santen Pharmaceutical Co., Japan). Acanthamoeba cells were counted in a hemocytometer and adjusted to a density of $1 \times 10^{5} / \mathrm{ml}$ in PYG medium. Pseudomonas aeruginosa was added to the Acanthamoeba suspension to obtain a final bacterial density of $3 \times 10^{5} / \mathrm{ml}$ (low density) or $1 \times 10^{8} / \mathrm{ml}$ (high density). The two organisms were co-incubated at $25^{\circ} \mathrm{C}$ for $2 \mathrm{~h}$ before corneal inoculation.

Animals. We used 24 healthy Japan albino rabbits aged 10-12 weeks and weighing 2.5-3.0 kg. Tokyo Medical University Ethics Committee approved this study involving rabbits. Rabbits used in this study were treated and maintained in rigid accordance to the ARVO Resolution on the Use of Animals in Research. All corneas were examined using a slit lamp before inoculation to exclude any abnormality.

Inoculation technique. Inoculation of Acanthamoeba alone or with $P$. aeruginosa was performed as described previously ${ }^{18}$. The right eye of each rabbit was tested and the left eye served as control. Briefly, general anesthesia was induced by intravenous injection of $0.5 \mathrm{ml} / \mathrm{kg}$ of $3 \%$ sodium pentobarbital, and one drop of benoxinate was applied to the right eye prior to inoculation. Under an operation microscope (Leica-M841, Germany), a $26 \mathrm{G}$ needle attached to a microliter syringe was advanced to the center of the cornea, and $30 \mu \mathrm{l}$ of a suspension containing $1 \times 10^{5}$ Acanthamoeba cells/ml with or without $P$. aeruginosa was injected into the corneal stroma.

Clinical evaluation and end point. The anterior segments of both eyes were examined by a slit-lamp in all rabbits, every day for 5-7 days after inoculation. Inflammatory findings were scored as described previously ${ }^{19}$. The clinical end point was defined as the development of fulminant keratitis in the rabbit cornea, or on day 5 after inoculation when the rabbit cornea remained clear during the study. When the clinical end point was reached, the rabbit was euthanized by an intravenous injection of sodium pentobarbital. The eyes were enucleated for examinations.

Comparison of infection by Acanthamoeba with low-density P. aeruginosa treated with $1.5 \%$ LVFX eye drop and 1.5\% LVFX + BSP eye drops. The effects of topical BSP in rabbit corneas infected by Acanthamoeba with a low density $\left(3 \times 10^{5} / \mathrm{ml}\right)$ of $P$. aeruginosa were examined in 4 eyes of 4 rabbits in each group. Rabbits inoculated with Acanthamoeba plus low-density P. aeruginosa were treated topically with $1.5 \%$ LVFX eye drops alone or 1.5\% LVFX + BSP eye drops starting immediately after inoculation on day 0, 3 times a day until day 5. In the third group, rabbit eyes inoculated with Acanthamoeba plus low-density P. aeruginosa were treated immediately with 1.5\% LVFX eye drops and then $12 \mathrm{~h}$ later with BSP eye drops, 3 times a day until day 5 .

Clinical evaluations were performed as described above. Rabbits were euthanized when the clinical end point was reached.

Comparison of infection by Acanthamoeba with high density $P$. aeruginosa treated with $1.5 \%$ LVFX eye and $1.5 \%$ LVFX + BSP eye drops. The effects of topical BSP eye drops in rabbit corneas infected by Acanthamoeba with a high density $\left(1 \times 10^{8} / \mathrm{ml}\right)$ of $P$. aeruginos $a$ were examined in 6 eyes of 6 rabbits in each group. Rabbits inoculated with Acanthamoeba plus high-density P. aeruginosa were treated with $1.5 \%$ LVFX eye drops or 1.5\% LVFX + BSP eye drops immediately after inoculation, 3 times a day until day 7. Clinical evaluations were performed as described above. The rabbits were euthanized when the clinical end point was reached.

Histological evaluation. The corneas of rabbits inoculated with Acanthamoeba and high-density P. aeruginosa treated with $1.5 \%$ LVFX + BSP eye drops were examined for the presence of Acanthamoeba. The cornea removed from the enucleated eye was mounted in embedding compound (Tissue-Tek OCT, Miles Scientific, Naperville, II) and snap-frozen in liquid nitrogen. Sections were cut at a thickness of $5 \mu \mathrm{m}$ on a cryostat at $-30^{\circ} \mathrm{C}$ and were mounted on polylysine-coated slides. All the sections were stained initially by Fungiflora Y and then by PAS. At least ten sections were examined for each eye. Histological localization of Acanthamoeba cysts was evaluated.

Statistical analysis. The data obtained were compiled into a database (Excel Microsoft Windows) and analyzed using statistical software (MedCalc). Mean and standard deviation for each group were calculated for analysis of quantitative variables, and Mann-Whitney tests were used to determine differences among groups. The significance level was set at $\mathrm{p}<0.05$.

\section{Data Availability}

The datasets generated during and/or analyzed during the current study are available from the corresponding author on reasonable request.

\section{References}

1. Chong, E. M. \& Dana, M. R. Acanthamoeba keratitis. Int. Ophthalmol. Clin. 47, 33-46 (2007).

2. Khan, N. A. Acanthamoeba: biology and increasing importance in human health. FEMS Microbiol. Rev. 30, 564-595 (2006).

3. Naginton, J. et al. Amoebic infection of the eye. Lancet 2, 1537-1540 (1974).

4. Duguid, I. G. et al. Outcome of acanthamoeba keratitis treated with polyhexamethyl biguanide and propamidine. Ophthalmology 104, 1587-1592 (1997). 
5. Park, D. H. et al. The role of topical corticosteroids in the management of Acanthamoeba keratitis. Cornea 16, 277-283 (1997).

6. Osato, M. S., Robinson, N. M., Wilhelmus, K. R. \& Jones, D. B. In vitro evaluation of antimicrobial compounds for cysticidal activity against Acanthamoeba. Rev. Infect. Dis. 13(Suppl 5), S431-435 (1991)

7. O'Day, D. M. \& Head, W. S. Advances in the management of keratomycosis and Acanthamoeba keratitis. Cornea 19, 681-687 (2000).

8. Robaei, D., Carnt, N., Minassian, D. C. \& Dart, J. K. The impact of topical corticosteroid use before diagnosis on the outcome of Acanthamoeba keratitis. Ophthalmology 121, 1383-1388 (2014).

9. McClellan, K., Howard, K., Niederkorn, J. Y. \& Alizadeh, H. Effect of steroids on Acanthamoeba cysts and trophozoites. Invest. Ophthalmol. Vis. Sci. 42, 2885-2893 (2001).

10. Tu, E. Y. et al. Polymicrobial keratitis: Acanthamoeba and infectious crystalline keratopathy. Am. J. Ophthalmol. 148, 13-19 e2 (2009).

11. Lee, B., Grossniklaus, H. E. \& Edelhauser, H. F. Concurrent acanthamoeba and Fusarium keratitis with silicone hydrogel contact lens use. Cornea 29, 210-213 (2010).

12. Iovieno, A., Ledee, D. R., Miller, D. \& Alfonso, E. C. Detection of bacterial endosymbionts in clinical acanthamoeba isolates. Ophthalmology 117(445-452), 52 e1-3 (2010).

13. Ikeda, Y. et al. Assessment of real-time polymerase chain reaction detection of Acanthamoeba and prognosis determinants of Acanthamoeba keratitis. Ophthalmology 119, 1111-1119 (2012).

14. Nakagawa, H. et al. Investigation of the role of bacteria in the development of Acanthamoeba keratitis. Cornea 34, 1308-1315 (2015).

15. Nakagawa, H. et al. Number of bacteria and time of coincubation with bacteria required for the development of Acanthamoeba keratitis. Cornea 36, 353-357 (2017).

16. Garate, M. et al. In vitro pathogenicity of Acanthamoeba is associated with the expression of the mannose-binding protein. Invest. Ophthalmol. Vis. Sci. 47, 1056-1062 (2006).

17. Institute CaLS. Methods for Dilution Antimicrobial Susceptibility Tests for Bacteria That Grow Aerobically; Approved StandardNinth Edition. CLSI document M07-A9 32, (2012).

18. Polat, Z. A., Ozcelik, S., Vural, A., Yildiz, E. \& Cetin, A. Clinical and histologic evaluations of experimental Acanthamoeba keratitis. Parasitol. Res. 101, 1621-1625 (2007).

19. Johnson, M. K. et al. The role of pneumolysin in ocular infections with Streptococcus pneumoniae. Curr. Eye Res. 9, 1107-1114 (1990).

\section{Acknowledgements}

This research received no specific grant from any funding agency in the public, commercial or not-for-profit sectors.

\section{Author Contributions}

H.N. conceived and designed the study. H.N., N.K., T.E .conducted the experiments. T.H., A.N., S.K. and H.G. analyzed and interpreted the data. H.N. wrote the manuscript. All authors reviewed the manuscript and approved of its submission.

\section{Additional Information}

Supplementary information accompanies this paper at https://doi.org/10.1038/s41598-019-49128-7.

Competing Interests: The authors declare no competing interests.

Publisher's note: Springer Nature remains neutral with regard to jurisdictional claims in published maps and institutional affiliations.

(c) (i) Open Access This article is licensed under a Creative Commons Attribution 4.0 International

License, which permits use, sharing, adaptation, distribution and reproduction in any medium or format, as long as you give appropriate credit to the original author(s) and the source, provide a link to the Creative Commons license, and indicate if changes were made. The images or other third party material in this article are included in the article's Creative Commons license, unless indicated otherwise in a credit line to the material. If material is not included in the article's Creative Commons license and your intended use is not permitted by statutory regulation or exceeds the permitted use, you will need to obtain permission directly from the copyright holder. To view a copy of this license, visit http://creativecommons.org/licenses/by/4.0/.

(C) The Author(s) 2019 